\title{
Fracture Behavior Transition in (001) Cracked Metal Nanoplates Induced by Surface Effect
}

\author{
Hokun Kimt, Duc Tam Ho ${ }^{2}$ and Sung Youb Kim ${ }^{1 *}$ \\ ${ }^{1}$ Department of Mechanical Engineering, Ulsan National Institute of Science and \\ Technology, Ulsan 44919, South Korea
}

2Physical Science and Engineering Division (PSE), King Abdullah University of Science and Technology (KAUST), Thuwal 23955-6900, Saudi Arabia.

*Corresponding author: sykim@unist.ac.kr

\section{Supporting Information}




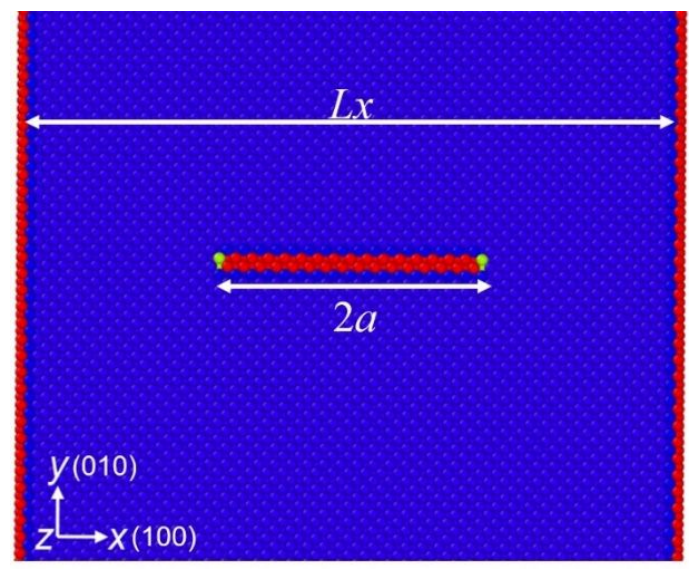

Figure S1. Schematic of cracked nanoplate used in this study.
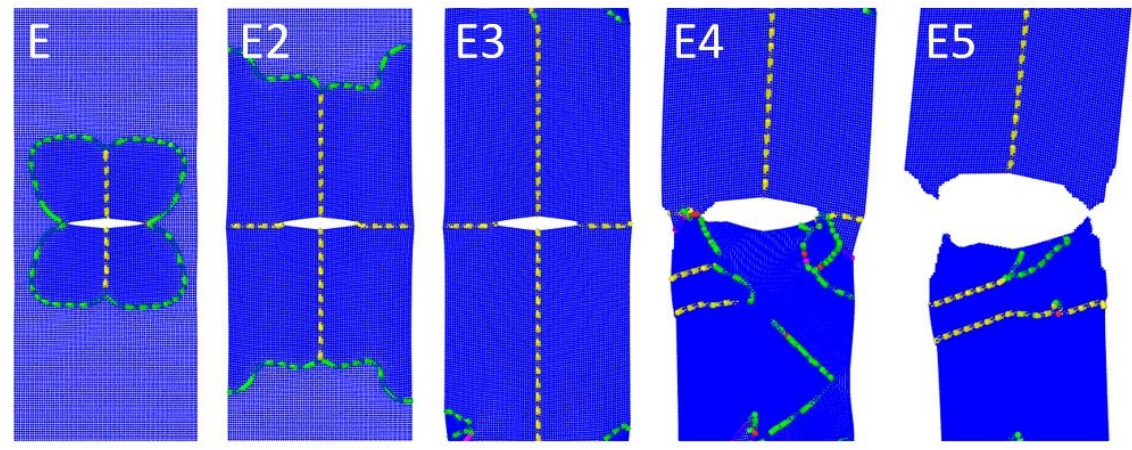

$$
\begin{aligned}
& \text { dislocation type } \\
& \text { other } \\
& 1 / 6<112>\text { (Shockley) } \\
& 1 / 6<110>\text { (Stair-rod) } \\
& 1 / 3<001>\text { (Hirth) }
\end{aligned}
$$

Figure. S2 The failure process of an elastic instability-dominant fracture for a cracked nanoplate with the dislocation type. E-E5 correspond to the points in the Figure 2(c) in the main text. The type of dislocation is obtained through DXA provided by OVITO'1. 


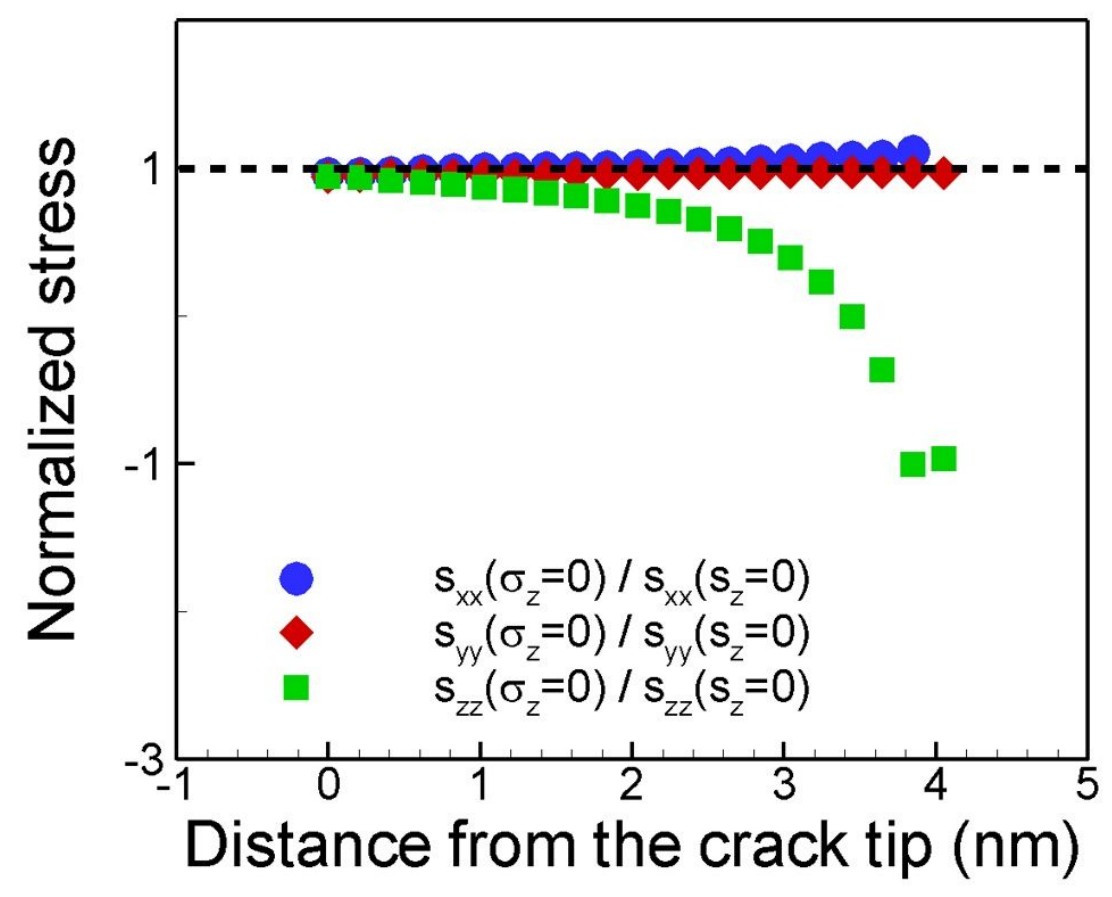

Figure S3 The stress distribution from the crack tip in the $x$-direction of a cracked nanoplate with and without a surface effect at a strain of 0.053 . To compare the stress field, we divide each stress component of a cracked nanoplate with a surface effect $\left(\sigma_{\mathrm{zz}}=0\right)$ by the corresponding stress component of a cracked nanoplate without a surface effect $\left(\mathrm{s}_{\mathrm{zz}}=0\right)$. The stress in the $x$ - and $y$ directions are almost the same. By contrast, the stress in the $z$-direction varies significantly as the distance from the crack tip increases. The value of $s_{z z}$ of a cracked nanoplate with the surface effect must be saturated to the value of $-2 \mathrm{f} / \mathrm{L}_{\mathrm{x}}$, whereas the value of $s_{z z}$ of a cracked nanoplate without a surface effect must be zero at sufficiently distant from the crack tip. Applying tensile stress in the $z$-direction removes the surface effect by preventing the cracked nanoplates from undergoing elastic instability. Thus, it changes the fracture behavior, but it does not alter the stress in the other directions. 


\section{Reference}

(1) Stukowski, A. Visualization and Analysis of Atomistic Simulation Data with OVITO-the Open Visualization Tool. Model. Simul. Mater. Sci. Eng. 2010, 18 (1), 015012 https://doi.org/10.1088/0965-0393/18/1/015012. 